\title{
Speckle observations of double stars with PISCO at Pic du Midi: measurements in 1998.
}

\author{
Scardia, M. \\ Osservatorio Astronomico di Brera, Via E. Bianchi 46, 22055 Merate, Italy \\ and \\ Prieur, J.-L. \\ UMR 5572 d'Astrophysique, Observatoire Midi-Pyrénées - Centre National de la Recherche Scientifique, \\ 14, Avenue Edouard Belin, 31400 Toulouse, France \\ and \\ Aristidi, E. \\ UMR 6525 Astrophysique, Université de Nice Sophia - Antipolis - Centre National de la Recherche \\ Scientifique, Parc Valrose, 06108 Nice Cedex 2, France \\ and \\ Koechlin, L. \\ UMR 5572 d'Astrophysique, Observatoire Midi-Pyrénées - Centre National de la Recherche Scientifique, \\ 14, Avenue Edouard Belin, 31400 Toulouse, France
}

\begin{abstract}
We present astrometric measurements of binary stars based on speckle observations of 164 independent sequences of observations $\left(\sim 10^{4}\right.$ frames each $)$ made with the PISCO speckle camera at Pic du Midi. They concern 147 objects whose duplicity was detected for 134 of them with a separation in the range $0.1-1.0^{\prime \prime}$. These objects were mainly selected among grade 3 orbits to improve the accuracy of their orbits and constrain their masses.

We discovered the binarity of $59 \mathrm{Aql}$ with an angular separation of $0.09 \pm 0.01^{\prime \prime}$.

Subject headings: Methods: data analysis - Techniques: interferometric - Binaries: close
\end{abstract}

\section{Introduction}

The aim of our program is to observe all the short period orbital couples which are well suited to the telescope and the instrumentation we are using (magnitude, declination and angular separation) and which have an orbit of grade 3 (see Worley et al. (1983) for the definition of the grade of an orbit, which is an indication of the quality of this orbit) so that a limited number of observations may improve this orbit to grade 2. Using Hipparcos parallaxes, these orbits will then allow a reasonably good determination of total system masses. This program has already provided a publication with measurements of 48 objects in 199597 and the revision of 8 orbits (Aristidi et al. 1999).

The observations were carried out in June and September 1998 with the PISCO $^{1}$ speckle camera (Prieur et al. 1994, 1998), developed by Observatoire Midi-Pyrénées and operated at ${ }^{1}$ PISCO stands for "Pupil Interferometry Speckle camera
and COronagraph". 
the Cassegrain Focus of the 2-meter Télescope Bernard Lyot (TBL) of the Pic du Midi Observatory. It is a remotely controlled versatile instrument that can be configured in various observing modes during the observations (e.g., imaging and spectroscopic modes) which provides a powerful tool for investigating the field of close binary stars as already shown in previous publications (Carbillet et al. 1996; Aristidi et al. 1997b, 1999). An overall precision better than $0.01^{\prime \prime}$ can be reached under normal or good observing conditions with an observing sequence of $\sim 5$ minutes.

New orbits have been updated for some of the objects presented here (e.g., ADS 161, ADS 221, ADS 281, ADS 1097, ADS 1522, ADS 1762, ADS 2034, ADS 2236 and ADS 10140) and will be presented in a forthcoming paper (Scardia et al. 2000). We also plan to perform photometric measurements of these observational data and present them in another paper, with additional data concerning stars with composite spectrum (Prieur et al. 2000).

\section{Observations}

The measurements described in the following are related to two observing runs in 1998: on June 12th, August 27th, 30th, 31st, September 3rd and 5 th. Seven more nights allocated to this program were completely spoiled because of bad weather. It was better than the previous runs: we could open the dome during $46 \%$ of the total allocated nights, whereas over the period 1993-1997 this rate was $33 \%$, with more than one hundred allocated nights for PISCO (the average TBL rate is $\sim 50 \%$ ).

The PISCO speckle camera was used in its full pupil imaging mode (Prieur et al. 1994, 1998). The atmospheric chromatic dispersion was corrected with real time computer-controlled Risley prisms, which provided a good correction with circular auto-correlation peaks (cf. section 3), except for a handful of objects due to a strong wind that caused an elongation of these peaks.

As a detector, we used the intensified CCD detector (Philips IP800T, herafter called ICCD) of Université de Nice which is described in Aristidi et al. (1997b). It generates a video output at a rate of 50 frames/sec which is recorded on a SVHS video tape.

\section{Data reduction}

The procedure we used to extract the astrometry parameters of the double stars is based on the analysis of the mean auto-correlation function of short exposured frames.

The data recorded on video cassettes were digitized by an electronic "Full RIO" PCI board made by Ellips (Ellips/Rio 1999) at a rate of 50 frames/sec with a specially designed program controlling a video-cassette-recorder developped by JLP in $\mathrm{C}++$. The digitized elementary frames were buffered into the memory of the PC (typically by sets of 200 frames) and processed to compute the mean auto-correlations and the mean crosscorrelation between two elementary frames separated by about one second (i.e., larger than the coherence time).

Following Worden et al. (1977), we subtracted the mean cross-correlation to the mean autocorrelation, which removed the (possibly strong) background of the mean auto-correlation and allowed an easy display of the secondary peaks of the auto-correlation of the binary star.

The center of the auto-correlation peaks was accurately determined with a specially-designed interactive program written by JLP and described in Prieur et al. (2000). Alternatively, for the closest binaries, the astrometric measurements were done in the Fourier plane by fitting a cosine function with a program developped by EA and JLP.

The orientation of the frames was calibrated using the tracks of stars obtained by moving the telescope along the declination and right ascension axes. The estimated uncertainty was $\pm 0.35^{\circ}$, which has been taken into account for estimating the error of the position angle measurements displayed in Table 4.

The magnification scale was obtained by measuring a calibrating grid that can be placed at the entrance focal plane of the speckle camera. Using the value of $50.4 \pm 0.1 \mathrm{~m}$ for the focal length of the TBL (determined by the Pic du Midi service of optics), we derived the values of respectively 0.0109 $\pm 0.0001,0.0258 \pm 0.0001$ and $0.1654 \pm 0.0005$ arc$\mathrm{sec} / \mathrm{pixel}$ for the three available magnifications obtained with a focal length of $10 \mathrm{~mm}, 20 \mathrm{~mm}$ and $50 \mathrm{~mm}$ for the imaging optics in our camera.

The validity of this calibration was checked 
both internally by observing the same object with a different magnification (thus checking the ratio of the magnification scales) and externally with "astrometric standards" (cf. Prieur et al. (2000)).

\section{Results and discussion}

The astrometric measurements are displayed in Table 4. For each observing sequence, we give the name of the object (col. \#1); the Focal Length $(\mathrm{FL})$ in $\mathrm{mm}$ of the eyepiece used as imaging lens in the magnification wheel; the filter (col. \#4) (as described in Table 2) ${ }^{2}$; the epoch (col. \#5) in Besselian years; the FWHM seeing (col. \#8) which was measured on the long integration obtained by adding all the elementary frames from that sequence; and the angular separation $\rho$ in arcseconds and the position angle $\theta$ in degrees.

The position angle of the companion $\theta$ (col. \#6 of Table 4) was measured relative to the North and increasing in the direction of increasing right ascension on the auto-correlation function, which leaves a $180^{\circ}$ ambiguity. In some cases, when the signal to noise ratio was good enough, the restricted triple-correlation technique described in Aristidi et al. (1997a) allowed us to remove this uncertainty and obtain absolute angle positions: this is noted with an asterisk in col. \#8.
${ }^{2}$ For WDS $18145+0011$, the filter value (V) is uncertain because of a problem with the filter wheel during this observation
In the last column, we give some more information:

- $(\mathrm{F})$ indicates that the measurement was done in the Fourier space by fitting a model to the fringes instead of measuring the position of the secondary peaks of the autocorrelation function. This was preferentially done for the closest binaries when the angular separation was less than $0.15^{\prime \prime}$ because it gives a better accuracy in this case.

- $(\mathrm{F}=)$ means that the two methods (Fourier and auto-correlation) gave exactly the same result.

- "Fuzzy", "faint contrast" are related to the secondary peaks of the auto-correlation function.

- "Artifacts close to the X axis": to mention the presence of some artifacts on the autocorrelation caused by the response of the ICCD to individual photons. In some cases the corresponding pattern was close to the secondary peaks of the binary and increased the measurement errors. When the artifacts were superimposed on these peaks (i.e. for $\theta \sim 100^{\circ}$ or $\theta \sim 280^{\circ}$ ), we used the Fourier fitting method.

- "Possible $180^{\circ}$ ambiguity": the quadrant determination (cf. previous paragraph) was noisy and the result is uncertain.

For WDS $16563+6502$, the two components were so widely separated that it was also possible to do a direct measurement on the long integration. 
TABLE 1

Relative astrometric data (epoch, $\rho, \theta$, Resp. in COl. \#5, \#7 and \#8) of the binary stars (SEe SeCtion 4). The POSition ANGLes $\theta$ HAVE a $180^{\circ}$ AMbiguity EXCEPT When ASTERISKED (For WHICH A TRIPLE CORRELATION METHOD REMOVED THIS AMBIGUITY).

\begin{tabular}{|c|c|c|c|c|c|c|c|c|}
\hline $\begin{array}{l}\text { WDS } \\
(1)\end{array}$ & $\begin{array}{c}\text { Name } \\
(2)\end{array}$ & $\begin{array}{c}\mathrm{FL} \\
(\mathrm{mm}) \\
(3)\end{array}$ & $\begin{array}{l}\text { Filt. } \\
(4)\end{array}$ & $\begin{array}{c}\text { Epoch } \\
\text { (5) }\end{array}$ & $\begin{array}{l}\text { Seeing } \\
\text { arcsec } \\
(6)\end{array}$ & $\begin{array}{c}\rho \\
\operatorname{arcsec} \\
(7)\end{array}$ & $\begin{array}{c}\theta \\
\text { degrees } \\
(8)\end{array}$ & $\begin{array}{l}\text { Comments } \\
\text { (9) }\end{array}$ \\
\hline $00121+5337$ & BU 1026 - ADS 148 & 10 & $\mathrm{R}$ & 1998.679 & 1.9 & $0.274 \pm 0.002$ & $296.9 \pm 0.5^{*}$ & \\
\hline $00134+2659$ & STT 2 - ADS 161 & 20 & $\mathrm{~V}$ & 1998.663 & 1.7 & $0.364 \pm 0.007$ & $171.0 \pm 0.6^{*}$ & $(\mathrm{~F}=)$ \\
\hline $00167+3629$ & STT 4 - ADS 221 & 10 & $\mathrm{R}$ & 1998.679 & 2.4 & $0.342 \pm 0.003$ & $138.8 \pm 0.5$ & \\
\hline $00173+0852$ & A 1803 - ADS 238 AB & 20 & $\mathrm{~V}$ & 1998.663 & 2.8 & $0.168 \pm 0.02$ & $123.2 \pm 1.5$ & Fuzzy $(\mathrm{F})$ \\
\hline$"$ & STF $22-$ ADS 238 AB-C & 20 & V & 1998.663 & 2.8 & - & - & Not resolved. \\
\hline $00182+7256$ & A $803-$ ADS 243 & 10 & $\mathrm{R}$ & 1998.665 & 3.0 & $0.233 \pm 0.003$ & $117.3 \pm 0.5$ & Very faint contrast. \\
\hline$"$ & $"$ & 20 & $\mathrm{~V}$ & 1998.665 & 3.7 & $0.232 \pm 0.006$ & $119.0 \pm 2.0$ & Artifacts close to $\mathrm{X}$ axis $(\mathrm{F})$. \\
\hline $00206+1219$ & BU 1015 - ADS 281 & 10 & $\mathrm{R}$ & 1998.679 & 2.8 & $0.423 \pm 0.003$ & $94.7 \pm 0.5$ & \\
\hline $00214+6700$ & STT 6 - ADS 293 & 20 & $\mathrm{~V}$ & 1998.665 & 3.6 & $0.618 \pm 0.003$ & $154.6 \pm 0.5^{*}$ & \\
\hline $00308+4732$ & BU 394 - ADS 416 & 10 & $\mathrm{~V}$ & 1998.666 & 2.8 & $0.180 \pm 0.002$ & $88.5 \pm 0.6$ & Artifacts close to $\mathrm{X}$ axis. \\
\hline " & " & 20 & $\mathrm{~V}$ & 1998.666 & 3.8 & $0.175 \pm 0.009$ & $82.0 \pm 1.0$ & \\
\hline $00318+5432$ & STT 12 - ADS 434 & 20 & $\mathrm{R}$ & 1998.665 & 3.8 & $0.400 \pm 0.003$ & $15.6 \pm 0.6$ & \\
\hline $00488+1842$ & BU 495 - ADS 673 & 20 & $\mathrm{~V}$ & 1998.663 & 1.9 & $0.158 \pm 0.008$ & $140.8 \pm 1.5$ & Fuzzy (F) \\
\hline $00516+2238$ & A 1808 - ADS 701 & 20 & $\mathrm{~V}$ & 1998.663 & 1.8 & $0.159 \pm 0.005$ & $12.4 \pm 2.0$ & Fuzzy (F) \\
\hline " & " & 10 & $\mathrm{~V}$ & 1998.663 & 2.4 & $0.159 \pm 0.002$ & $11.9 \pm 0.8$ & 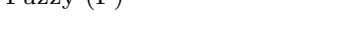 \\
\hline $00532+0406$ & A 2307 - ADS 732 & 10 & $\mathrm{R}$ & 1998.679 & 2.4 & $0.181 \pm 0.002$ & $59.4 \pm 0.6^{*}$ & Possible $180^{\circ}$ ambiguity. \\
\hline $00546+1912$ & STT 20 - ADS 746 & 20 & $\mathrm{~V}$ & 1998.663 & 2.5 & $0.518 \pm 0.003$ & $14.6 \pm 0.5$ & \\
\hline $00550+2338$ & STF 73 - ADS 755 & 20 & V & 1998.663 & 1.8 & $0.871 \pm 0.003$ & $305.7 \pm 0.5^{*}$ & \\
\hline $00583+2124$ & BU $302-A D S 805$ & 10 & $\mathrm{R}$ & 1998.679 & 2.2 & $0.362 \pm 0.002$ & $184.8 \pm 0.5^{*}$ & \\
\hline $01011+6022$ & A 926 - ADS 832 & 20 & $\mathrm{~V}$ & 1998.666 & 4.4 & $0.382 \pm 0.003$ & $154.8 \pm 0.6$ & \\
\hline $01093+4715$ & STT 515 - ADS 940 & 20 & RL & 1998.666 & 3.6 & $0.505 \pm 0.004$ & $127.1 \pm 0.5^{*}$ & \\
\hline $01137+0733$ & STF 100 - ADS 996 AB & 20 & $\mathrm{~V}$ & 1998.655 & 3.1 & - & - & Not resolved. \\
\hline$"$ & BU 1029 - ADS 996 BC & 20 & $\mathrm{~V}$ & 1998.655 & 3.1 & - & - & Not resolved. \\
\hline $01213+1132$ & BU 4 - ADS 1097 & 10 & $\mathrm{R}$ & 1998.679 & 2.4 & $0.544 \pm 0.003$ & $111.7 \pm 0.5^{*}$ & \\
\hline $01297+2250$ & A 1910 - ADS 1183 & 10 & $\mathrm{R}$ & 1998.679 & 2.2 & $0.123 \pm 0.002$ & $35.7 \pm 1.2$ & \\
\hline $01443+5732$ & BU 870 - ADS 1359 & 20 & $\mathrm{~V}$ & 1998.655 & 3.4 & $0.747 \pm 0.003$ & $348.2 \pm 0.5^{*}$ & \\
\hline " & & 20 & RL & 1998.666 & 3.6 & $0.744 \pm 0.003$ & $348.6 \pm 0.5^{*}$ & \\
\hline $01512+2439$ & HO 311 - ADS 1473 & 10 & $\mathrm{R}$ & 1998.679 & 2.7 & $0.247 \pm 0.003$ & $164.8 \pm 0.5$ & \\
\hline $01551+2847$ & STF 183 - ADS 1522 & 10 & $\mathrm{R}$ & 1998.679 & 2.7 & $0.329 \pm 0.002$ & $156.6 \pm 0.5$ & \\
\hline $01558+0151$ & STF 186 - ADS 1538 & 20 & $\mathrm{R}$ & 1998.679 & 2.2 & $1.046 \pm 0.005$ & $61.5 \pm 0.5$ & \\
\hline $01570+3101$ & A 819 - ADS 1548 & 10 & $\mathrm{R}$ & 1998.679 & 2.5 & $0.198 \pm 0.002$ & $73.3 \pm 0.5$ & \\
\hline $02020+0246$ & STF $202-$ ADS 1615 & 20 & $\mathrm{R}$ & 1998.679 & 2.5 & $1.863 \pm 0.005$ & $274.3 \pm 0.5^{*}$ & \\
\hline $02037+2556$ & STF 208 - ADS 1631 & 20 & $\mathrm{R}$ & 1998.679 & 2.5 & $1.159 \pm 0.005$ & $334.1 \pm 0.5^{*}$ & \\
\hline $02157+2503$ & COU 79 & 20 & V & 1998.663 & 2.5 & - & - & Not resolved. \\
\hline " & " & 20 & $\mathrm{~W}$ & 1998.663 & 2.5 & - & - & Not resolved. \\
\hline $02158+0638$ & A 2013 - ADS 1729 & 10 & $\mathrm{R}$ & 1998.679 & 3.4 & - & - & Not resolved. \\
\hline $02182+3920$ & A 207 - ADS 1762 & 10 & $\mathrm{R}$ & 1998.679 & 1.7 & $0.317 \pm 0.002$ & $354.8 \pm 0.5^{*}$ & \\
\hline $02257+6133$ & STF 257 - ADS 1833 & 20 & $\mathrm{~V}$ & 1998.655 & 4.1 & $0.391 \pm 0.003$ & $63.7 \pm 0.5^{*}$ & \\
\hline $02290+6724$ & CHR $6 \mathrm{Aa}-\mathrm{ADS} 1860$ & 20 & V & 1998.655 & 3.2 & - & - & Not resolved. \\
\hline " & " & 10 & $\mathrm{~V}$ & 1998.666 & 2.2 & $0.299 \pm 0.002$ & $91.9 \pm 0.7$ & Artifacts close to $\mathrm{X}$ axis. \\
\hline$"$ & $"$ & 20 & RL & 1998.666 & 3.9 & $0.30 \pm 0.05$ & $93.0 \pm 3.0^{*}$ & Very faint contrast. \\
\hline $02333+5218$ & STT 42 - ADS 1938 & 20 & RL & 1998.666 & 3.6 & 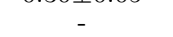 & - & Cloudy, not resolved. \\
\hline $02407+2637$ & STT 43 - ADS 2034 & 20 & $\mathrm{R}$ & 1998.679 & 3.6 & $0.754 \pm 0.003$ & $354.9 \pm 0.5^{*}$ & \\
\hline $02572+0153$ & A 2413 - ADS 2236 & 10 & $\mathrm{R}$ & 1998.680 & 2.4 & $0.418 \pm 0.003$ & $140.5 \pm 0.5$ & \\
\hline $03048+5330$ & LAB - ADS 2324 & 10 & $\mathrm{~V}$ & 1998.666 & 1.6 & $0.251 \pm 0.002$ & $244.8 \pm 0.5^{*}$ & \\
\hline$"$ & $"$ & 20 & $\mathrm{~V}$ & 1998.666 & 3.1 & $0.253 \pm 0.008$ & $245.1 \pm 1.5^{*}$ & $(\mathrm{~F})$ \\
\hline $03127+7133$ & STT 50 - ADS 2377 & 20 & $\mathrm{~V}$ & 1998.655 & 3.4 & $1.102 \pm 0.005$ & $158.2 \pm 0.5$ & Slightly elongated. \\
\hline $03175+6540$ & STT 52 - ADS 2436 & 20 & V & 1998.655 & 3.5 & $0.481 \pm 0.003$ & $63.5 \pm 0.5^{*}$ & \\
\hline " & " & 20 & $\mathrm{~V}$ & 1998.666 & 3.2 & $0.480 \pm 0.003$ & $64.0 \pm 0.5$ & \\
\hline $03284+6015$ & A 980 - ADS 2538 & 10 & V & 1998.666 & 2.5 & $0.336 \pm 0.003$ & $171.6 \pm 0.5$ & \\
\hline$"$ & & 20 & $\mathrm{~V}$ & 1998.666 & 3.2 & $0.331 \pm 0.003$ & $171.4 \pm 0.5$ & \\
\hline $03350+6002$ & STF 400 - ADS 2612 & 20 & V & 1998.666 & 3.0 & $1.453 \pm 0.005$ & $265.7 \pm 0.5^{*}$ & \\
\hline $03344+2428$ & STF 412 - ADS 2616 & 20 & $\mathrm{R}$ & 1998.679 & 1.2 & $0.684 \pm 0.004$ & $357.8 \pm 0.5^{*}$ & \\
\hline $03496+6318$ & MOAI 1 - SAO 12917 & 20 & $\mathrm{~V}$ & 1998.654 & 3.4 & $0.082 \pm 0.004$ & $158.3 \pm 1.5$ & Faint \\
\hline $05167+4600$ & ANJ 1 - ADS 3841 & 10 & B & 1998.666 & 1.9 & $0.052 \pm 0.002$ & $241.3 \pm 1.5$ & (F) Capella \\
\hline$"$ & $"$ & 10 & $\mathrm{~V}$ & 1998.666 & 2.0 & $0.050 \pm 0.002$ & $242.3 \pm 1.5$ & (F) \\
\hline $13202+1747$ & A 2166 - ADS 8863 & 20 & $\mathrm{R}$ & 1998.446 & 2.4 & - & - & Not resolved. \\
\hline $13519+1008$ & B 2543 & 20 & $\mathrm{R}$ & 1998.446 & 2.2 & - & - & Not resolved. \\
\hline $14153+0308$ & STF 1819 - ADS 9182 & 20 & $\mathrm{R}$ & 1998.446 & 2.9 & $0.896 \pm 0.005$ & $23.0 \pm 0.5$ & \\
\hline $14268+1625$ & A 2069 - ADS 9264 & 20 & $\mathrm{R}$ & 1998.446 & 2.3 & $0.268 \pm 0.008$ & $40.2 \pm 0.5$ & $(\mathrm{~F})$ \\
\hline $15161-0454$ & STF 3091 - ADS 9557 & 20 & $\mathrm{R}$ & 1998.446 & 2.8 & $0.584 \pm 0.004$ & $49.4 \pm 0.5$ & \\
\hline $15273+1738$ & A 2074 - ADS 9645 & 20 & $\mathrm{R}$ & 1998.446 & 3.3 & - & - & Video gain too low. \\
\hline
\end{tabular}


TABLE 1-Continued

\begin{tabular}{|c|c|c|c|c|c|c|c|c|}
\hline $\begin{array}{l}\text { WDS } \\
(1)\end{array}$ & $\begin{array}{c}\text { Name } \\
(2)\end{array}$ & $\begin{array}{c}\mathrm{FL} \\
(\mathrm{mm}) \\
(3)\end{array}$ & $\begin{array}{l}\text { Filt. } \\
(4)\end{array}$ & $\begin{array}{c}\text { Epoch } \\
\text { (5) }\end{array}$ & $\begin{array}{l}\text { Seeing } \\
\operatorname{arcsec} \\
(6)\end{array}$ & $\begin{array}{c}\rho \\
\operatorname{arcsec} \\
(7)\end{array}$ & $\begin{array}{c}\theta \\
\text { degrees } \\
(8)\end{array}$ & $\begin{array}{c}\text { Comments } \\
\text { (9) }\end{array}$ \\
\hline$"$ & $"$ & 20 & $\mathrm{R}$ & 1998.446 & 2.4 & $0.241 \pm 0.008$ & $253.4 \pm 1.0^{*}$ & Artifacts close to $\mathrm{X}$ axis $(\mathrm{F})$. \\
\hline $15282+0251$ & A 2175 - ADS 9654 & 20 & $\mathrm{R}$ & 1998.446 & 2.4 & $0.258 \pm 0.007$ & $22.4 \pm 0.7$ & $(\mathrm{~F}=)$ \\
\hline $15328+1945$ & HU 577 - ADS 9692 & 20 & $\mathrm{R}$ & 1998.446 & 2.4 & $0.274 \pm 0.006$ & $185.0 \pm 0.5$ & $(\mathrm{~F}=)$ \\
\hline $15542+1659$ & A 2080 - ADS 9831 & 20 & $\mathrm{R}$ & 1998.446 & 2.4 & $0.350 \pm 0.008$ & $97.0 \pm 0.9$ & Artifacts close to $\mathrm{X}$ axis $(\mathrm{F})$. \\
\hline $16367+6948$ & BU 953 - ADS 10140 & 10 & $\mathrm{R}$ & 1998.678 & 1.6 & $0.280 \pm 0.002$ & $87.2 \pm 1.7^{*}$ & Elongated. \\
\hline $16458-0046$ & A 1141 - ADS 10196 & 20 & $\mathrm{R}$ & 1998.446 & 2.5 & $0.195 \pm 0.008$ & $24.3 \pm 2.0$ & Fuzzy (F) \\
\hline $16511+0925$ & STF 2106 - ADS 10229 & 20 & $\mathrm{R}$ & 1998.446 & 2.4 & $0.683 \pm 0.005$ & $176.7 \pm 0.5^{*}$ & \\
\hline $16515+0113$ & STT 315 - ADS 10230 & 20 & $\mathrm{R}$ & 1998.446 & 2.5 & $0.496 \pm 0.003$ & $321.9 \pm 0.5^{*}$ & \\
\hline $\begin{array}{c}16563+6502 \\
"\end{array}$ & STF 2118 - ADS 10279 & 20 & $\mathrm{R}$ & 1998.678 & 1.8 & $\begin{array}{c}1.116 \pm 0.006 \\
1.07 \pm 0.02\end{array}$ & $\begin{array}{l}68.1 \pm 0.6^{*} \\
69.6 \pm 1.0^{*}\end{array}$ & $\begin{array}{l}\text { (auto-correlation) } \\
\text { (long integration) }\end{array}$ \\
\hline $17003+3056$ & HR 6324 & 20 & $\mathrm{~V}$ & 1998.654 & 3.0 & - & - & Not resolved. \\
\hline $17082-0105$ & A 1145 - ADS 10355 & 20 & $\mathrm{R}$ & 1998.446 & 2.3 & $0.590 \pm 0.005$ & $357.2 \pm 0.5^{*}$ & \\
\hline $17088+6543$ & STA 1 - HR 6396 & 10 & OIII & 1998.679 & 1.6 & - & - & Not resolved. \\
\hline $17303-0103$ & STF 2173 - ADS 10598 & 20 & $\mathrm{R}$ & 1998.446 & 2.4 & $0.686 \pm 0.005$ & $322.8 \pm 0.5^{*}$ & \\
\hline $17366+0722$ & A 1156 - ADS 10659 & 20 & $\mathrm{R}$ & 1998.446 & 2.2 & $0.193 \pm 0.008$ & $174.0 \pm 1.0$ & Fuzzy $(\mathrm{F}=)$ \\
\hline 17399-0039 & BU 631 - ADS 10696 & 20 & $\mathrm{R}$ & 1998.446 & 2.5 & $0.213 \pm 0.015$ & $279.6 \pm 1.0^{*}$ & Artifacts close to $\mathrm{X}$ axis $(\mathrm{F})$. \\
\hline $17505+0715$ & STT 337 - ADS 10828 & 20 & $\mathrm{R}$ & 1998.446 & 2.1 & $0.484 \pm 0.004$ & $171.6 \pm 0.5$ & \\
\hline $17564+1820$ & STF 2245 - ADS 10905 Aa-B & 20 & $\mathrm{~V}$ & 1998.654 & 2.0 & $2.638 \pm 0.005$ & $112.5 \pm 0.5^{*}$ & \\
\hline $17564+1820$ & MCA 49 - ADS 10905 Aa & 20 & $\mathrm{~V}$ & 1998.654 & 2.0 & - & - & Not resolved. \\
\hline $17564+1820$ & - ADS 10905 B & 20 & $\mathrm{~V}$ & 1998.654 & 2.0 & - & - & Not resolved. \\
\hline $17571+0004$ & STF 2244 - ADS 10912 & 20 & $\mathrm{R}$ & 1998.673 & 2.2 & $0.532 \pm 0.004$ & $277.2 \pm 0.5^{*}$ & Possible $180^{\circ}$ ambiguity. \\
\hline $17572+2400$ & MCA 50 & 10 & $\mathrm{~V}$ & 1998.673 & 2.3 & - & - & Not resolved. \\
\hline $18063+3824$ & HU 1186 - ADS 11071 & 10 & $\mathrm{R}$ & 1998.673 & 2.9 & - & - & Not resolved. \\
\hline$"$ & $"$ & 20 & $\mathrm{R}$ & 1998.673 & 3.0 & $0.362 \pm 0.009$ & $110.1 \pm 0.5$ & \\
\hline $18078+2606$ & CHR $67 \mathrm{Aa}$ & 20 & $\mathrm{~V}$ & 1998.654 & 3.3 & $0.245 \pm 0.006$ & $335.3 \pm 1.5^{*}$ & Very faint \\
\hline $18102+1628$ & STF 2289 - ADS 11123 & 20 & $\mathrm{R}$ & 1998.673 & 2.4 & $1.221 \pm 0.008$ & $220.8 \pm 0.5^{*}$ & \\
\hline $18117+3327$ & B 2545 - ADS 11149 AB & 20 & $\mathrm{~V}$ & 1998.654 & 3.0 & - & - & Not resolved. \\
\hline $18117+3327$ & HO 82 - ADS 11149 AB-C & 20 & $\mathrm{~V}$ & 1998.654 & 3.0 & $0.711 \pm 0.005$ & $219.4 \pm 0.5^{*}$ & \\
\hline $18145+0011$ & STF 2294 - ADS 11186 & 20 & V? & 1998.673 & 3.1 & $1.279 \pm 0.005$ & $273.9 \pm 0.5^{*}$ & Possible $180^{\circ}$ ambiguity. \\
\hline $18197+1016$ & HU 197 - ADS 11260 & 20 & $\mathrm{R}$ & 1998.673 & 2.8 & $0.445 \pm 0.004$ & $86.7 \pm 0.5$ & \\
\hline $18207+7120$ & STT 353 - ADS 11311 & 10 & $\mathrm{R}$ & 1998.679 & 1.5 & $0.429 \pm 0.003$ & $271.4 \pm 0.5^{*}$ & Slightly elongated. \\
\hline $18250-0135$ & AC 11 - ADS 11324 & 20 & $\mathrm{R}$ & 1998.673 & 3.1 & $0.884 \pm 0.005$ & $175.6 \pm 0.5$ & \\
\hline $18250+2723$ & STF 2315 - ADS 11334 & 20 & $\mathrm{R}$ & 1998.673 & 3.3 & $0.656 \pm 0.005$ & $124.3 \pm 0.5$ & \\
\hline $18338+1744$ & HU 322 - ADS 11454 AB & 20 & $\mathrm{~V}$ & 1998.654 & 3.0 & $0.115 \pm 0.006$ & $71.6 \pm 1.5$ & Artifacts close to $\mathrm{X}$ axis. \\
\hline $18338+1744$ & WAK 21 - ADS 11454 CD & 20 & $\mathrm{~V}$ & 1998.654 & 3.0 & $0.409 \pm 0.003$ & $261.7 \pm 0.5^{*}$ & \\
\hline $18338+1744$ & STF 2339 - ADS 11454 AB-CD & 20 & $\mathrm{~V}$ & 1998.654 & 3.0 & $1.675 \pm 0.007$ & $277.0 \pm 0.5^{*}$ & \\
\hline $18340+5221$ & A 1377 - ADS 11468 & 10 & $\mathrm{~V}$ & 1998.679 & 1.5 & $0.258 \pm 0.002$ & $113.4 \pm 0.5$ & \\
\hline $18355+2336$ & STT 359 - ADS 11479 & 20 & $\mathrm{R}$ & 1998.673 & 2.2 & $0.711 \pm 0.005$ & $7.5 \pm 0.5^{*}$ & \\
\hline $18410+2450$ & A 2988 - ADS 11574 & 10 & $\mathrm{R}$ & 1998.673 & 3.1 & - & - & Not resolved. \\
\hline $18413+3018$ & STF 2367 - ADS 11579 & 10 & $\mathrm{R}$ & 1998.673 & 1.9 & $0.305 \pm 0.002$ & $79.9 \pm 0.5^{*}$ & \\
\hline $18462+6412$ & HU 937 - ADS 11692 & 20 & $\mathrm{R}$ & 1998.679 & 2.0 & $0.937 \pm 0.005$ & $329.5 \pm 0.5^{*}$ & Slightly elongated. \\
\hline $18466+3821$ & HU 1191 - ADS 11680 & 10 & $\mathrm{R}$ & 1998.673 & 1.9 & $0.156 \pm 0.002$ & $40.5 \pm 0.6$ & \\
\hline $18477+4904$ & HEI 72 & 10 & $\mathrm{~V}$ & 1998.679 & 1.5 & $0.580 \pm 0.004$ & $227.7 \pm 0.5^{*}$ & \\
\hline $18575+5814$ & STF 2438 - ADS 11897 & 20 & $\mathrm{R}$ & 1998.679 & 1.8 & $0.862 \pm 0.005$ & $0.7 \pm 0.5^{*}$ & Slightly elongated. \\
\hline $19022+5216$ & HU 757 - ADS 11979 & 10 & W & 1998.679 & 1.7 & $0.139 \pm 0.005$ & $17.5 \pm 1.2$ & \\
\hline $19106+5429$ & A 1391 - ADS 12144 & 10 & $\mathrm{~W}$ & 1998.679 & 1.5 & $0.139 \pm 0.007$ & $153.6 \pm 1.5$ & Very fuzzy. (F) \\
\hline $19180+2012$ & COU 321 & 10 & $\mathrm{~V}$ & 1998.679 & 1.8 & $0.087 \pm 0.005$ & $132.8 \pm 3.0$ & $(\mathrm{~F}=)$ \\
\hline$"$ & $"$ & 10 & $\mathrm{~W}$ & 1998.679 & 3.3 & - & - & Very fuzzy, doubtful. \\
\hline $19216+5223$ & BU 1129 - ADS 12366 & 10 & $\mathrm{~V}$ & 1998.679 & 1.7 & $0.234 \pm 0.007$ & $169.2 \pm 0.5$ & \\
\hline $19296+1224$ & A 1653 - ADS 12515 & 10 & $\mathrm{R}$ & 1998.673 & 2.3 & $0.213 \pm 0.009$ & $170.0 \pm 1.5$ & Faint autoc. peaks (F) \\
\hline $19411+1349$ & KUI 93 & 20 & V & 1998.654 & 2.9 & $0.193 \pm 0.009$ & $133.3 \pm 1.5$ & Artifacts close to $\mathrm{X}$ axis. \\
\hline $19542+0828$ & HR $7595-59$ Aql & 20 & $\mathrm{R}$ & 1998.657 & 2.9 & $0.09 \pm 0.01$ & $60 \pm 5$ & New double \\
\hline $19573+0513$ & A 604 - ADS 13156 & 10 & $\mathrm{R}$ & 1998.679 & 2.1 & $0.133 \pm 0.003$ & $50.6 \pm 1.2$ & \\
\hline $19580+0456$ & A 606 - ADS 13169 & 10 & $\mathrm{R}$ & 1998.679 & 2.4 & $0.548 \pm 0.004$ & $147.9 \pm 0.5$ & \\
\hline $20158+2749$ & MCA $60 \mathrm{Aa}$ & 20 & $\mathrm{~V}$ & 1998.654 & 3.0 & $0.239 \pm 0.006$ & $143.9 \pm 1.2^{*}$ & \\
\hline $20232+2052$ & A 288 - ADS 13777 & 10 & $\mathrm{~V}$ & 1998.679 & 2.3 & $0.160 \pm 0.005$ & $86.8 \pm 0.8$ & \\
\hline $20290+0710$ & A 610 - ADS 13894 & 10 & $\mathrm{R}$ & 1998.679 & 2.9 & $0.377 \pm 0.004$ & $49.5 \pm 0.5$ & Very faint autoc. peaks. \\
\hline $20311+3332$ & COU 1962 & 10 & $\mathrm{R}$ & 1998.679 & 2.1 & - & - & Not resolved. \\
\hline $20311+1548$ & A 1675 - ADS 13944 & 20 & $\mathrm{R}$ & 1998.662 & 2.6 & - & - & Not resolved. \\
\hline$"$ & & 10 & V & 1998.662 & 3.2 & - & - & Not resolved. \\
\hline $20375+1436$ & BU 151 - ADS 14073 & 20 & $\mathrm{~V}$ & 1998.662 & 2.4 & $0.453 \pm 0.004$ & $334.0 \pm 0.5^{*}$ & \\
\hline $20396+0458$ & KUI 99 & 20 & $\mathrm{~V}$ & 1998.662 & 3.1 & $0.381 \pm 0.005$ & $124.4 \pm 1.2$ & Artifacts close to $\mathrm{X}$ axis. \\
\hline
\end{tabular}




\begin{tabular}{|ccc|}
\hline Name & $\begin{array}{c}\text { Central wavelength } \\
(\mathrm{nm})\end{array}$ & $\begin{array}{c}\text { Bandwith } \\
(\mathrm{nm})\end{array}$ \\
\hline $\mathrm{B}$ & 447 & 47 \\
OIII & 501 & 11 \\
V & 530 & 57 \\
R & 644 & 70 \\
RL & 743 & 69 \\
W & 650 & 418 \\
\hline
\end{tabular}

Table 2: Characteristics of the filters used (cf. col \#4 of Table 4). "W" means "White" since no filter has been used. In that case, the bandwidth selection was done with the ICCD response only.

The smallest errors for the angular separation were estimated at $\sim 0.002^{\prime \prime}$ for the best cases on the basis of the uncertainties coming from the determination of the center of the auto-correlation peak and those affecting the scale determination. Similarily we found $\sim 0.5^{\circ}$ for the angle position.

To investigate the possibility of systematic errors (e.g. a possible bad value for the focal length of the telescope), we plotted the residuals relative to the ephemerides computed with the known orbits for these objets (Fig. 1). The residuals are mostly concentrated near the point $(\Delta \theta=0 ; \Delta \rho=$ 0 ) with no clear offset, which is a good indication that our measurements have not systematic errors. This test was also successful for the last two sets of observations with PISCO published in Aristidi et al. (1997b) and Aristidi et al. (1999).

When we examine the case of the largest residuals, we see that they correspond to old orbits: Chara 6Aa (Heintz 1962), Fox 102 (Popovic, 1972), A 1391 (Baize, 1987), STT 483 (Valbousquet, 1981), Hei 72 (Baize, 1983) and STF 2244 (Baize, 1984).

For the same objects, more recent orbits, when available, lead to a better agreement:

- STT 483: $\Delta \theta=-3.7^{\circ}, \rho=0.03^{\prime \prime}$ with (Heintz, 1996),

- STF 2244: $\Delta \theta=0.1^{\circ}, \rho=0.04^{\prime \prime}$ with (Heintz, 1997).

The case of Cou 321 is more complex: it is difficult to observe (separation less than $0.1^{\prime \prime}$ ) and since the first measurements in 1969 it has not yet covered half of its orbit, with a big gap of observations between 1972 and 1985 (cf. Fig. 4 of Aristidi et al. (1999)). Hence even the most recent orbits (Docobo, 1998, Aristidi, 1999) should be considered as preliminary. The residuals of table 4 are larger than the errors of our observations $\left(\sigma_{\theta}=3\right.$. and $\left.\sigma_{\rho}=0.005\right)$ although the orbits are recent:

- $\Delta \theta=8.7^{\circ}, \rho=+0.02^{\prime \prime}$ with (Docobo, 1998).

- $\Delta \theta=-0.5^{\circ}, \rho=-0.07^{\prime \prime}$ with our revised orbit (Aristidi, 1999).

This example clearly shows that (i) it is essential to have a continuous program of measurements and (ii) more speckle observations of good quality are needed to determine this orbit. 
TABLE 1-Continued

\begin{tabular}{|c|c|c|c|c|c|c|c|c|}
\hline $\begin{array}{l}\text { WDS } \\
(1)\end{array}$ & $\begin{array}{c}\text { Name } \\
(2)\end{array}$ & $\begin{array}{c}\mathrm{FL} \\
(\mathrm{mm}) \\
(3)\end{array}$ & $\begin{array}{l}\text { Filt. } \\
(4)\end{array}$ & $\begin{array}{c}\text { Epoch } \\
\text { (5) }\end{array}$ & $\begin{array}{l}\text { Seeing } \\
\text { arcsec } \\
(6)\end{array}$ & $\begin{array}{c}\rho \\
\operatorname{arcsec} \\
(7)\end{array}$ & $\begin{array}{c}\theta \\
\text { degrees } \\
(8)\end{array}$ & $\begin{array}{l}\text { Comments } \\
\text { (9) }\end{array}$ \\
\hline $20397+1556$ & WCK 2 - ADS 14121 & 20 & $\mathrm{~V}$ & 1998.662 & 2.5 & $0.144 \pm 0.015$ & $125.8 \pm 2.0^{*}$ & Faint $(\mathrm{F})$ \\
\hline $20449+1219$ & STF 2723 - ADS 14233 & 20 & $\mathrm{~V}$ & 1998.654 & 3.4 & $1.072 \pm 0.005$ & $131.8 \pm 0.5^{*}$ & \\
\hline $20451+1244$ & BU 64 - ADS 14238 & 20 & $\mathrm{~V}$ & 1998.662 & 3.0 & $0.680 \pm 0.005$ & $168.4 \pm 0.5$ & \\
\hline $20572+4110$ & $\nu \mathrm{Cyg}$ & 20 & V & 1998.654 & 3.3 & - & - & Not resolved. \\
\hline " & $" 10$ & 10 & $\mathrm{~V}$ & 1998.679 & 1.6 & - & - & Not resolved. \\
\hline $21002+0731$ & KUI 102 & 20 & $\mathrm{~V}$ & 1998.662 & 2.6 & $0.360 \pm 0.003$ & $13.8 \pm 0.5$ & \\
\hline $21080+0509$ & STT 527 - ADS 14666 & 20 & $\mathrm{~V}$ & 1998.663 & 2.6 & $0.307 \pm 0.005$ & $120.0 \pm 2.0$ & $\begin{array}{l}\text { Faint autoc. peaks, } \\
\text { Artifacts close to } \mathrm{X} \text { axis. }(\mathrm{F})\end{array}$ \\
\hline $21125+2821$ & HO 152 - ADS 14748 & 10 & $\mathrm{R}$ & 1998.679 & 2.5 & $0.211 \pm 0.002$ & $114.5 \pm 0.6$ & \\
\hline $21135+0713$ & BU 270 - ADS 14759 & 20 & $\mathrm{~W}$ & 1998.679 & 2.0 & $0.611 \pm 0.005$ & $349.9 \pm 0.5^{*}$ & \\
\hline $21135+1559$ & HU 767 - ADS 14761 & 20 & $\mathrm{~V}$ & 1998.663 & 2.4 & $0.214 \pm 0.003$ & $140.9 \pm 2.3$ & Fuzzy $(\mathrm{F}=)$ \\
\hline $21423+0554$ & HU 280 - ADS 15236 & 20 & $\mathrm{~V}$ & 1998.663 & 2.2 & $0.204 \pm 0.008$ & $158.3 \pm 1.1$ & $(\mathrm{~F}=)$ \\
\hline $21459+1153$ & A 1223 - ADS 15300 & 10 & $\mathrm{R}$ & 1998.679 & 2.9 & $0.151 \pm 0.008$ & $21.0 \pm 0.9$ & Faint autoc. peaks. \\
\hline $21555+1053$ & BU 75 - ADS 15447 & 20 & $\mathrm{R}$ & 1998.679 & 2.4 & $0.767 \pm 0.005$ & $15.6 \pm 0.5^{*}$ & \\
\hline $22077+2622$ & COU 537 & 20 & $\mathrm{~V}$ & 1998.663 & 2.4 & - & - & Not resolved. \\
\hline $22139+3944$ & MCA $70 \mathrm{Ab}-\mathrm{ADS} 15758$ & 20 & $\mathrm{~V}$ & 1998.654 & 3.0 & $0.390 \pm 0.003$ & $15.9 \pm 0.7$ & \\
\hline $22241-0451$ & BU 172 - ADS 15902 & 10 & V & 1998.679 & 2.1 & $0.269 \pm 0.002$ & $67.3 \pm 0.5^{*}$ & \\
\hline$"$ & " & 20 & $\mathrm{~V}$ & 1998.679 & 2.0 & $0.273 \pm 0.004$ & $66.5 \pm 0.8^{*}$ & \\
\hline 22288-0002 & STF 2909 - ADS 15971 & 20 & $\mathrm{~V}$ & 1998.679 & 2.1 & $1.935 \pm 0.005$ & $10.1 \pm 0.5^{*}$ & \\
\hline $22299+0425$ & STF 2912 - ADS 15988 & 20 & $\mathrm{~V}$ & 1998.663 & 1.8 & $0.413 \pm 0.003$ & $116.7 \pm 0.6^{*}$ & \\
\hline $22307+1758$ & COU 234 & 20 & $\mathrm{~V}$ & 1998.663 & 2.4 & - & - & Not resolved. \\
\hline $22359+3938$ & CHR 112 - ADS 16095 Aa & 20 & $\mathrm{~V}$ & 1998.654 & 3.9 & - & - & Not resolved. \\
\hline $22359+3938$ & STF 2922 - ADS 16095 Aa-B & 20 & $\mathrm{~V}$ & 1998.654 & 3.9 & - & - & Not resolved. \\
\hline $22385+0218$ & HO 479 - ADS 16131 & 20 & $\mathrm{~V}$ & 1998.663 & 2.5 & $0.465 \pm 0.04$ & $90.0 \pm 1.5^{*}$ & Artifacts close to $\mathrm{X}$ axis. $(\mathrm{F})$ \\
\hline $22402+3731$ & HO 188 - ADS 16164 & 10 & $\mathrm{R}$ & 1998.679 & 2.2 & $0.373 \pm 0.003$ & $35.5 \pm 0.5$ & \\
\hline $22405+0631$ & HU 494 - ADS 16165 & 10 & $\mathrm{R}$ & 1998.679 & 3.3 & - & - & Not resolved. \\
\hline $22408-0333$ & KUI 114 & 20 & $\mathrm{~V}$ & 1998.663 & 2.2 & $0.300 \pm 0.003$ & $130.9 \pm 0.5$ & $(\mathrm{~F}=)$ \\
\hline $22485+3106$ & BU 1146 - ADS 16278 & 10 & $\mathrm{R}$ & 1998.679 & 2.1 & $0.222 \pm 0.002$ & $89.2 \pm 0.5^{*}$ & Possible $180^{\circ}$ ambiguity. \\
\hline $22514+2624$ & HO 482 - ADS 16314 & 20 & $\mathrm{~V}$ & 1998.663 & 2.2 & $0.443 \pm 0.003$ & $24.8 \pm 0.5$ & \\
\hline $22570+2441$ & COU 542 & 10 & $\mathrm{R}$ & 1998.679 & 2.4 & $0.237 \pm 0.002$ & $168.8 \pm 0.5$ & \\
\hline $22585+0922$ & STT 536 - ADS 16417 & 20 & $\mathrm{~V}$ & 1998.663 & 2.9 & $0.280 \pm 0.006$ & $167.0 \pm 0.7$ & \\
\hline $22592+1144$ & STT 483 - ADS 16428 & 20 & $\mathrm{~V}$ & 1998.663 & 1.7 & $0.503 \pm 0.005$ & $333.1 \pm 0.5^{*}$ & \\
\hline $23088+1057$ & A 1238 - ADS 16539 & 10 & $\mathrm{R}$ & 1998.679 & 2.1 & $0.299 \pm 0.002$ & $147.8 \pm 0.5$ & \\
\hline $23176+1819$ & HU 400 - ADS 16650 & 20 & V & 1998.663 & 2.2 & $0.358 \pm 0.003$ & $100.4 \pm 1.0$ & Artifacts close to $\mathrm{X}$ axis. $(\mathrm{F}=)$ \\
\hline $23189+0525$ & BU 80 - ADS 16665 & 10 & $\mathrm{R}$ & 1998.679 & 2.4 & $0.272 \pm 0.002$ & $19.4 \pm 0.5$ & \\
\hline $23340+3120$ & BU 720 - ADS 16836 & 20 & $\mathrm{~V}$ & 1998.663 & 1.6 & $0.546 \pm 0.004$ & $93.0 \pm 0.5^{*}$ & \\
\hline $23374+0737$ & FOX 102 - ADS 16873 & 10 & $\mathrm{R}$ & 1998.679 & 2.4 & $0.191 \pm 0.002$ & $355.5 \pm 0.5$ & \\
\hline $23431+1150$ & A 1242 - ADS 16951 & 20 & $\mathrm{R}$ & 1998.679 & 2.8 & $0.981 \pm 0.006$ & $152.8 \pm 0.5$ & \\
\hline $23440+2922$ & AGC 14 - ADS 16957 & 20 & V & 1998.663 & 1.6 & $0.835 \pm 0.005$ & $266.9 \pm 0.5^{*}$ & \\
\hline $23498+2740$ & A 424 - ADS 17030 & 20 & $\mathrm{~V}$ & 1998.663 & 1.9 & $0.164 \pm 0.006$ & $137.6 \pm 0.5^{*}$ & $(\mathrm{~F})$ \\
\hline 23529-0309 & FIN 359 & 10 & $\mathrm{R}$ & 1998.679 & 2.1 & - & - & Not resolved. \\
\hline $23568+0443$ & A 2100 - ADS 17111 & 10 & $\mathrm{R}$ & 1998.679 & 2.0 & $0.213 \pm 0.002$ & $288.2 \pm 0.5^{*}$ & \\
\hline
\end{tabular}


TABLE 3

RESIDUALS OF THE EXPECTED POSITIONS COMPUTED WITH KNOWN ORBITS (OBSERVED - COMPUTED) (CF. SECTION 4).

\begin{tabular}{|c|c|c|c|c|c|}
\hline WDS & Name & Orbit & \multicolumn{2}{|c|}{ Residuals O-C } & Comments \\
\hline $00121+5337$ & BU 1026 - ADS 148 & Hartkopf (1996) & 0.00 & -0.1 & \\
\hline $00134+2659$ & STT 2 - ADS 161 & Heintz (1979) & 0.04 & 5.4 & \\
\hline $00167+3629$ & STT 4 - ADS 221 & Scardia (1982) & -0.08 & -13.1 & \\
\hline $00173+0852$ & A 1803 - ADS 238 & Baize (1987) & 0.02 & -1.8 & \\
\hline \multirow[t]{2}{*}{$00182+7256$} & A 803 - ADS 243 & Zulevic (1996) & 0.01 & -6.1 & $\mathrm{R} 10 \mathrm{~mm}$ \\
\hline & & Zulevic (1996) & 0.01 & -4.4 & $\mathrm{~V} 20 \mathrm{~mm}$ \\
\hline $00206+1219$ & BU 1015 - ADS 281 & Scardia (1980) & -0.00 & -3.2 & \\
\hline $00214+6700$ & STT 6 - ADS 293 & Muller (1954) & -0.10 & 5.0 & \\
\hline $00308+4732$ & BU 394 - ADS 416 & Zulevic (1997) & -0.05 & -3.8 & \\
\hline $00318+5432$ & STT 12 - ADS 434 & Heintz (1995) & -0.06 & 0.7 & \\
\hline $00488+1842$ & BU 495 - ADS 673 & Couteau (1989) & -0.03 & -8.3 & \\
\hline \multirow[t]{4}{*}{$00516+2238$} & A 1808 - ADS 701 & Docobo (1988) & -0.01 & -2.0 & $\mathrm{~V} 20 \mathrm{~mm}$ \\
\hline & & Docobo (1988) & -0.01 & -2.5 & $\mathrm{~V} 10 \mathrm{~mm}$ \\
\hline & & Baize (1989) & 0.01 & 3.9 & V $20 \mathrm{~mm}$ \\
\hline & & Baize (1989) & 0.01 & 3.4 & $\mathrm{~V} 10 \mathrm{~mm}$ \\
\hline \multirow[t]{2}{*}{$00532+0406$} & A 2307 - ADS 732 & Walker (1975) & -0.01 & -2.6 & \\
\hline & & Heintz (1975) & -0.08 & 2.1 & \\
\hline $00546+1912$ & STT 20 - ADS 746 & Couteau (1984) & 0.03 & 2.2 & \\
\hline $00550+2338$ & STF 73 - ADS 755 & Docobo (1989) & -0.00 & -0.3 & \\
\hline \multirow{2}{*}{$00583+2124$} & BU 302 - ADS 805 & Popovic (1997) & -0.03 & 3.7 & \\
\hline & & Zulevic (1997) & -0.07 & 5.0 & \\
\hline $01011+6022$ & A 926 - ADS 832 & Popovic (1997) & -0.03 & 2.0 & \\
\hline $01093+4715$ & STT 515 - ADS 940 & Baize (1958) & 0.04 & -0.4 & \\
\hline $01213+1132$ & BU 4 - ADS 1097 & Muller (1953) & 0.08 & 7.4 & \\
\hline $01297+2250$ & A 1910 - ADS 1183 & Hartkopf (1996) & 0.00 & 1.0 & \\
\hline \multirow[t]{2}{*}{$01443+5732$} & BU 870 - ADS 1359 & Popovic (1995) & 0.08 & 2.7 & $\mathrm{~V} 20 \mathrm{~mm}$ \\
\hline & & Popovic (1995) & 0.08 & 3.1 & RL $20 \mathrm{~mm}$ \\
\hline $01512+2439$ & HO 311 - ADS 1473 & Hartkopf (1989) & 0.01 & 0.3 & \\
\hline $01551+2847$ & STF 183 - ADS 1522 & Couteau (1973) & -0.02 & 3.4 & \\
\hline $01558+0151$ & STF 186 - ADS 1538 & Mourao (1977) & 0.03 & -1.4 & \\
\hline $01570+3101$ & A 819 - ADS 1548 & Heintz (1997) & 0.01 & -4.6 & \\
\hline $02020+0246$ & STF 202 - ADS 1615 & Scardia (1983) & 0.03 & 1.8 & \\
\hline \multirow[t]{2}{*}{$02037+2556$} & STF 208 - ADS 1631 & Kranjc (1960) & -0.08 & -2.9 & \\
\hline & & Heintz (1996) & -0.08 & -2.9 & \\
\hline \multirow[t]{2}{*}{$02182+3920$} & A 207 - ADS 1762 & Heintz (1975) & -0.02 & -6.1 & \\
\hline & & Scardia (1982) & -0.05 & 3.4 & \\
\hline \multirow{2}{*}{$02257+6133$} & STF 257 - ADS 1833 & Zaera de & & & \\
\hline & & Toledo (1985) & -0.01 & 2.5 & \\
\hline \multirow[t]{2}{*}{$02290+6724$} & CHARA 6 Aa & Heintz (1962) & 0.21 & 49.7 & V 10mm \\
\hline & & Heintz (1962) & 0.21 & 50.8 & $\mathrm{RL} 20 \mathrm{~mm}$ \\
\hline $02407+2637$ & STT 43 - ADS 2034 & Heintz (1962) & -0.21 & -3.1 & \\
\hline \multirow[t]{2}{*}{$02572+0153$} & A 2413 - ADS 2236 & Scardia (1980) & -0.01 & 5.8 & \\
\hline & & Heintz (1991) & 0.01 & -2.7 & \\
\hline \multirow[t]{2}{*}{$03048+5330$} & LAB - ADS 2324 & McAlister (1982) & -0.02 & 2.4 & $\mathrm{~V} 10 \mathrm{~mm}$ \\
\hline & & McAlister (1982) & -0.02 & 2.7 & $\mathrm{~V} 20 \mathrm{~mm}$ \\
\hline $03127+7133$ & STT 50 - ADS 2377 & Popovic (1972) & 0.18 & 4.1 & \\
\hline \multirow[t]{2}{*}{$03175+6540$} & STT 52 - ADS 2436 & Heintz unpub. & 0.05 & 2.2 & V 20mm 1998.655 \\
\hline & & Heintz unpub. & 0.05 & 2.7 & V 20mm 1998.666 \\
\hline \multirow[t]{4}{*}{$03284+6015$} & A 980 - ADS 2538 & Zulevic (1992) & 0.02 & 2.0 & V $10 \mathrm{~mm}$ \\
\hline & & Zulevic (1992) & 0.02 & 1.8 & $\mathrm{~V} 20 \mathrm{~mm}$ \\
\hline & & Baize (1993) & 0.01 & -0.8 & $\mathrm{~V} 10 \mathrm{~mm}$ \\
\hline & & Baize (1993) & 0.01 & -1.0 & $\mathrm{~V} 20 \mathrm{~mm}$ \\
\hline
\end{tabular}


TABle 3-Continued

\begin{tabular}{|c|c|c|c|c|c|}
\hline WDS & Name & Orbit & \multicolumn{2}{|c|}{ Residuals O-C } & Comments \\
\hline \multirow{2}{*}{$03350+6002$} & STF 400 - ADS 2612 & Baize (1952) & -0.12 & 2.9 & \\
\hline & & Scardia (1981) & 0.01 & -0.4 & \\
\hline \multirow{2}{*}{$03344+2428$} & STF 412 - ADS 2616 & Luyten (1934) & -0.06 & -2.5 & \\
\hline & & Scardia (1985) & 0.03 & 2.5 & \\
\hline \multirow{2}{*}{$05167+4600$} & ANJ 1 - ADS 3841 & McAlister (1981) & 0.01 & -0.4 & \\
\hline & & McAlister (1981) & 0.01 & 0.6 & \\
\hline \multirow[t]{2}{*}{$14153+0308$} & STF 1819 - ADS 9182 & Houser (1987) & 0.01 & -0.5 & \\
\hline & & Baize (1987) & 0.08 & 7.3 & \\
\hline \multirow[t]{2}{*}{$14268+1625$} & A 2069 - ADS 9264 & Eggen (1965) & 0.03 & 4.7 & \\
\hline & & Couteau (1986) & -0.01 & 4.3 & \\
\hline \multirow[t]{2}{*}{$15161-0454$} & STF 3091 - ADS 9557 & Laques (1971) & 0.10 & 5.5 & \\
\hline & & Mason (1999) & 0.00 & 2.1 & \\
\hline $15273+1738$ & A 2074 - ADS 9645 & Baize (1976) & 0.03 & 2.9 & \\
\hline $15282+0251$ & A 2175 - ADS 9654 & Heintz (1996) & 0.02 & -0.9 & \\
\hline $15328+1945$ & HU 577 - ADS 9692 & Couteau (1984) & 0.01 & 1.8 & \\
\hline \multirow{2}{*}{$15542+1659$} & A 2080 - ADS 9831 & Zulevic (1973) & 0.05 & -20.4 & \\
\hline & & Baize (1986) & 0.13 & -13.1 & \\
\hline $16367+6948$ & BU 953 - ADS 10140 & Scardia (1984) & 0.07 & 6.6 & \\
\hline \multirow[t]{2}{*}{$16458-0046$} & A 1141 - ADS 10196 & Baize (1976) & 0.01 & 2.3 & \\
\hline & & Heintz (1982) & 0.00 & 5.6 & \\
\hline $16511+0925$ & STF 2106 - ADS 10229 & Heintz (1963) & 0.00 & 4.0 & \\
\hline $16515+0113$ & STT 315 - ADS 10230 & Docobo (1991) & 0.00 & 1.7 & \\
\hline \multirow[t]{2}{*}{$16563+6502$} & STF 2118 - ADS 10279 & Scardia (1981) & -0.14 & -0.1 & (auto-correlation) \\
\hline & & Scardia (1981) & -0.19 & 1.4 & (long integration) \\
\hline $17082-0105$ & A 1145 - ADS 10355 & Heintz (1995) & 0.11 & 3.2 & \\
\hline $17303-0103$ & STF 2173 - ADS 10598 & Heintz (1994) & 0.00 & 0.2 & \\
\hline $17366+0722$ & A 1156 - ADS 10659 & Docobo (1991) & -0.01 & 3.8 & \\
\hline \multirow[t]{2}{*}{ 17399-0039 } & BU 631 - ADS 10696 & Baize (1991) & -0.02 & 6.5 & \\
\hline & & Heintz (1996) & -0.01 & 3.2 & \\
\hline $17505+0715$ & STT 337 - ADS 10828 & Docobo $(1990)$ & 0.02 & 1.3 & \\
\hline \multirow{2}{*}{$17571+0004$} & STF 2244 - ADS 10912 & Baize (1984) & 0.34 & -13.6 & \\
\hline & & Heintz (1997) & 0.04 & 0.1 & \\
\hline \multirow[t]{2}{*}{$18063+3824$} & HU 1186 - ADS 11071 & Baize (1992) & 0.04 & 1.0 & \\
\hline & & Heintz (1995) & 0.05 & 0.2 & \\
\hline $18102+1628$ & STF 2289 - ADS 11123 & Hopmann (1964) & -0.02 & 2.8 & \\
\hline $18145+0011$ & STF 2294 - ADS 11186 & Luyten (1934) & 0.11 & 0.3 & \\
\hline \multirow{2}{*}{$18197+1016$} & HU 197 - ADS 11260 & Baize (1972) & 0.05 & 11.9 & \\
\hline & & Heintz (1995) & 0.03 & 1.7 & \\
\hline $18207+7120$ & STT 353 - ADS 11311 & Olevic (1990) & 0.03 & 2.3 & \\
\hline $18250-0135$ & AC 11 - ADS 11324 & Heintz (1995) & 0.02 & 0.4 & \\
\hline $18250+2723$ & STF 2315 - ADS 11334 & Heintz (1960) & -0.13 & 0.5 & \\
\hline $18340+5221$ & A 1377 - ADS 11468 & Scardia (1984) & 0.00 & -1.6 & \\
\hline $18355+2336$ & STT 359 - ADS 11479 & Symms (1964) & 0.02 & 1.3 & \\
\hline $18413+3018$ & STF 2367 - ADS 11579 & Cester (1991) & -0.02 & 0.0 & \\
\hline $18462+6412$ & HU 937 - ADS 11692 & Baize (1989) & 0.16 & -2.9 & \\
\hline $18466+3821$ & HU 1191 - ADS 11680 & Morel (1969) & -0.01 & -0.3 & \\
\hline $18477+4904$ & HEI 72 & Baize (1983) & 0.29 & -23.3 & \\
\hline $18575+5814$ & STF 2438 - ADS 11897 & Jastrzebski (1959) & -0.07 & 0.7 & \\
\hline $19022+5216$ & HU 757 - ADS 11979 & Heintz (1978) & 0.03 & -19.4 & \\
\hline $19106+5429$ & A 1391 - ADS 12144 & Baize (1987) & -0.03 & 22.3 & \\
\hline \multirow{2}{*}{$19180+2012$} & COU 321 & Docobo (1998) & 0.02 & 8.7 & \\
\hline & & Aristidi (1999) & -0.07 & -0.5 & \\
\hline
\end{tabular}




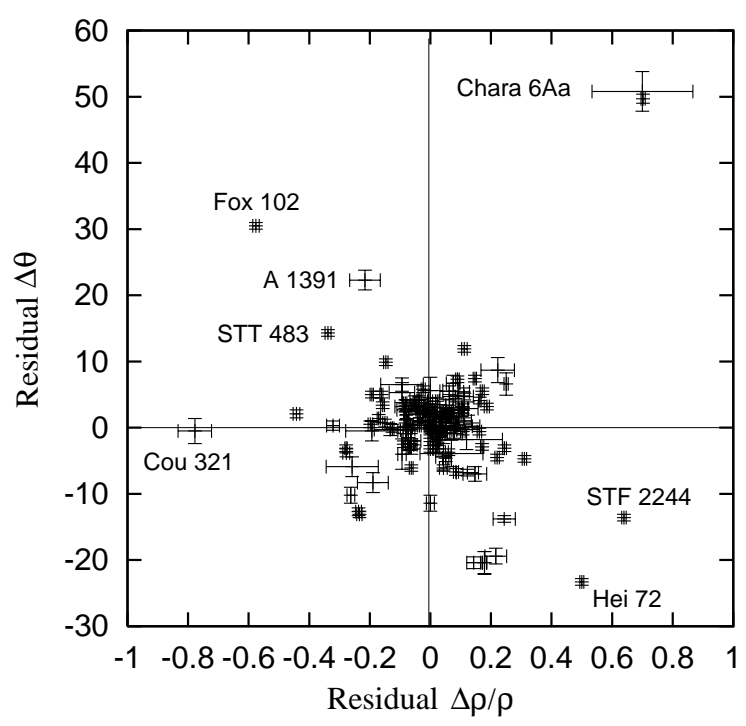

Fig. 1.- Residuals of the expected positions computed with known orbits (observed - computed) (cf. section 4).

\section{The new binary 59 Aql}

When reducing these data, it appeared that HR 7595, one of our reference stars, was actually double. Its power spectrum with the $\mathrm{R}$ filter exhibit clear fringes (cf. Fig. 2). The fringes are not so clear in $\mathrm{V}$, whereas nothing can be seen in B. The cosine fit in the Fourier space lead to: $\rho=0.09 \pm 0.01^{\prime \prime}$ and $\theta=60 . \pm 5 .^{\circ}$. The errors are rather large because the seeing conditions were rather poor $\left(\mathrm{FWHM}=2.9^{\prime \prime}\right)$. We encourage other observers to confirm this binarity.

HR 7595 (HD 188310, or 59 Aql) is not known as double in any of the data bases that we consulted. It is a bright ( $\mathrm{V}=4.71)$, G9III-type nearby star with a parallax of 16.0 mas.

\section{Conclusion}

The PISCO speckle camera of Observatoire Midi-Pyrénées with the ICCD of Université de Nice has proven to be well adapted to efficiently observe binary and multiple stars. Due to better weather conditions than in 1995-1997 (cf. Aristidi et al. (1999)), we have been able to provide a substancial number of measurements: 134 objects

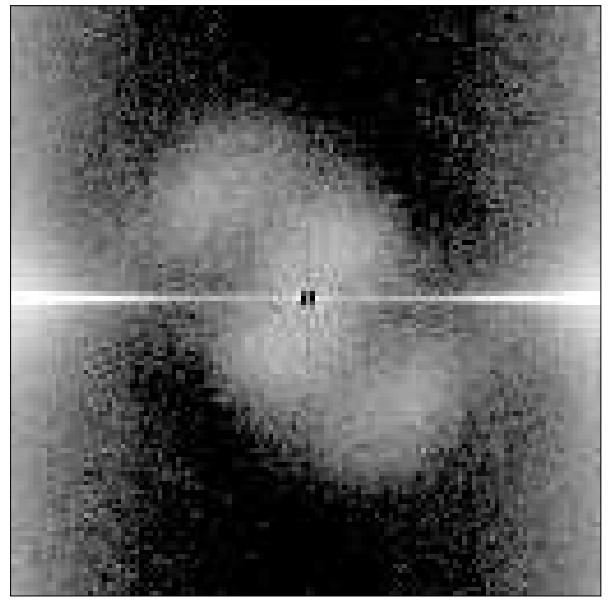

Fig. 2.- Modulus of the Fourier Transform of the new binary 59 Aql which exhibit clear fringes ( $\mathrm{R}$ filter).

compared to 48 in our previous paper, with an accuracy generally better than $0.01^{\prime \prime}$ for the angular separation and less than $1^{\circ}$ for the position angle.

We have discovered the binarity of $59 \mathrm{Aql}$ when examining the stars which were used as reference star for de-convolution, but the atmospheric conditions were rather poor with a FWHM of 2.9" and we encourage other observers to confirm this binarity.

The example of Cou 321 shows that such extensive observing programs with speckle cameras with medium-size telescopes are essential for a good orbit determination. Unfortunately the new policy of telescope time allocation is going to another direction. No other observing run has been allocated since 1998 and the observations reported in this paper will be probably the last of this programme.

\section{Acknowledgements:}

We would like to thank the technical staff of the "Télescope Bernard Lyot" for providing assistance during the observations.

We also thank Laurent Deynis (Student) and Gérard Daigne (Bordeaux Observatory, France) who participated to the observations respectively in June and September 1998. 
TABle 3-Continued

\begin{tabular}{|c|c|c|c|c|c|}
\hline \multirow[t]{2}{*}{ WDS } & \multirow[t]{2}{*}{ Name } & \multirow[t]{2}{*}{ Orbit } & \multicolumn{2}{|c|}{ Residuals O-C } & \multirow[t]{2}{*}{ Comments } \\
\hline & & & $\rho(\operatorname{arcsec})$ & $\theta($ degrees $)$ & \\
\hline $19216+5223$ & BU 1129 - ADS 12366 & Baize (1984) & -0.01 & 2.5 & \\
\hline $19296+1224$ & A 1653 - ADS 12515 & Heintz (1963) & -0.02 & 5.3 & \\
\hline $19573+0513$ & A 604 - ADS 13156 & Heintz (1991) & 0.01 & 6.7 & \\
\hline $19580+0456$ & A 606 - ADS 13169 & Baize (1984) & 0.17 & -4.7 & \\
\hline \multirow[t]{2}{*}{$20232+2052$} & A 288 - ADS 13777 & Docobo (1992) & 0.01 & 1.5 & \\
\hline & & Baize (1992) & 0.01 & 5.5 & \\
\hline $20290+0710$ & A 610 - ADS 13894 & Heintz (1979) & 0.02 & 2.5 & \\
\hline $20375+1436$ & BU 151 - ADS 14073 & Hartkopf (1989) & 0.02 & -0.4 & \\
\hline \multirow[t]{2}{*}{$20396+0458$} & KUI 99 & Baize (1981) & 0.00 & -11.4 & \\
\hline & & Heintz (1984) & -0.10 & -10.2 & \\
\hline $20397+1556$ & WCK 2 - ADS 14121 & Hartkopf (1989) & 0.01 & -3.9 & \\
\hline \multirow[t]{2}{*}{$20451+1244$} & BU 64 - ADS 14238 & Baize (1957) & 0.15 & -4.5 & \\
\hline & & Heintz (1995) & 0.11 & -0.6 & \\
\hline $21002+0731$ & KUI 102 & Heintz (1996) & 0.03 & 4.4 & \\
\hline $21080+0509$ & STT 527 - ADS 14666 & Popovic (1995) & 0.01 & 1.5 & \\
\hline $21125+2821$ & HO 152 - ADS 14748 & Baize (1981) & -0.05 & -12.7 & \\
\hline $21135+0713$ & BU 270 - ADS 14759 & Heintz (1979) & -0.00 & 0.0 & \\
\hline $21135+1559$ & HU 767 - ADS 14761 & Hartkopf (1989) & -0.02 & -4.0 & \\
\hline $21423+0554$ & HU 280 - ADS 15236 & Baize (1987) & 0.03 & -7.0 & \\
\hline $21459+1153$ & A 1223 - ADS 15300 & Couteau (1963) & -0.02 & -0.3 & \\
\hline \multirow{2}{*}{$21555+1053$} & BU 75 - ADS 15447 & Baize (1974) & -0.01 & 2.6 & \\
\hline & & Heintz (1996) & -0.00 & 0.4 & \\
\hline \multirow[t]{2}{*}{$22241-0451$} & BU 172 - ADS 15902 & Heintz (1996) & -0.01 & 4.1 & $\mathrm{~V} 10 \mathrm{~mm}$ \\
\hline & & Heintz (1996) & -0.01 & 3.3 & $\mathrm{~V} 20 \mathrm{~mm}$ \\
\hline 22288-0002 & STF 2909 - ADS 15971 & Heintz (1984) & 0.03 & 2.5 & \\
\hline $22299+0425$ & STF 2912 - ADS 15988 & Zulevic (1988) & -0.03 & -0.9 & \\
\hline \multirow[t]{2}{*}{$22385+0218$} & HO 479 - ADS 16131 & Zulevic (1979) & -0.09 & -0.5 & \\
\hline & & Heintz (1997) & -0.12 & -5.9 & \\
\hline $22402+3731$ & HO 188 - ADS 16164 & Docobo (1986) & 0.04 & -0.6 & \\
\hline \multirow[t]{2}{*}{$22408-0333$} & KUI 114 & Baize (1976) & -0.01 & 1.5 & \\
\hline & & Griffin (1987) & -0.02 & 0.5 & \\
\hline $22485+3106$ & BU 1146 - ADS 16278 & Couteau (1989) & -0.02 & -0.4 & \\
\hline \multirow[t]{2}{*}{$22514+2624$} & HO 482 - ADS 16314 & Morel (1970) & -0.00 & 3.1 & \\
\hline & & Starikova (1982) & -0.00 & 2.3 & \\
\hline $22570+2441$ & COU 542 & Couteau (1993) & 0.02 & -6.7 & \\
\hline $22585+0922$ & STT 536 - ADS 16417 & Cester (1991) & -0.09 & 0.3 & \\
\hline \multirow[t]{2}{*}{$22592+1144$} & STT 483 - ADS 16428 & Valbousquet (1981) & -0.17 & 14.3 & \\
\hline & & Heintz (1996) & 0.03 & -3.7 & \\
\hline $23088+1057$ & A 1238 - ADS 16539 & Muller (1955) & -0.01 & 1.5 & \\
\hline $23176+1819$ & HU 400 - ADS 16650 & Heintz (1962) & 0.02 & -0.8 & \\
\hline $23189+0525$ & BU 80 - ADS 16665 & Heintz (1996) & -0.04 & 9.9 & \\
\hline \multirow[t]{2}{*}{$23340+3120$} & BU 720 - ADS 16836 & Baize (1976) & 0.01 & -3.2 & \\
\hline & & Starikova (1982) & 0.01 & -2.0 & \\
\hline $23374+0737$ & FOX 102 - ADS 16873 & Popovic (1972) & -0.11 & 30.5 & \\
\hline $23431+1150$ & A 1242 - ADS 16951 & Zulevic (1977) & 0.24 & -3.1 & \\
\hline $23440+2922$ & AGC 14 - ADS 16957 & Popovic (1997) & -0.03 & 0.3 & \\
\hline \multirow{2}{*}{$23498+2740$} & A 424 - ADS 17030 & Heintz (1984) & 0.04 & -13.8 & \\
\hline & & Baize (1984) & 0.02 & -6.8 & \\
\hline $23568+0443$ & A 2100 - ADS 17111 & Heintz (1996) & 0.00 & 0.4 & \\
\hline
\end{tabular}




\section{REFERENCES}

Aristidi, E., Carbillet, M., Lyon, J.-F., Aime, C., 1997a, Astron. \& Astrophys Suppl., 125, 139.

Aristidi, E., Carbillet, M., Prieur, J.-L, Lopez, B., Bresson, Y., Koechlin, L., 1997b, Astron. \& Astrophys Suppl., 126, 555-561.

Aristidi, E., Prieur, J.-L., Scardia, M., Koechlin, L., Avila, R., Lopez, B., Rabbia, Y., Carbillet, M., Nisenson, P., Gezari, D., 1999, Astron. \& Astrophys Suppl., 134, 545-552.

Carbillet, M., Lopez, B., Aristidi, E., Bresson, Y., Aime, C., Ricort, G., Prieur, J.-L., Koechlin, L., Helmer, G., Lefèvre, J., Cruzalebes, P., 1996, Astron. \& Astrophys., 314, 112.

Ellips/Rio 1999: see the web page: "http://www.ellips.nl/rio.html".

Prieur, J.-L., Oblak, E., Lampens, P., Aristidi, E., Ruymaekers, G., Kurpinska-Winiarska, M., Koechlin, L., 2000, preprint.

Prieur, J.-L., Festou, M.C, Koechlin, L., André, C., Coll. National de Planétologie de l'INSU, Toulouse, 13-16 Juin 1994, p S1622 .

Prieur, J.-L, Koechlin, L., André, C., Gallou, G., Lucuix, C., 1998, Experimental Astronomy, vol 8, Issue 4, p 297-315.

Prieur, J.-L, Carquillat, J.-M., Ginestet, N., Koechlin, L., Scardia, M., Aristidi, E., 2000, in preparation.

Scardia, M., Prieur, J.-L, Aristidi, E., 2000, preprint.

Worden S.P., Murray K.S., Schmidt G.D., Angel J.R.P., 1977, Icarus 32, 450.

Worley, C.E., Heintz, W.D., 1983, U.S. Naval Obs. Publ. vol. XXIV, 7.

This 2-column preprint was prepared with the AAS LATEX macros v5.0. 